\title{
The implications for advanced maternal age on pregnancy complications and adverse neonatal outcomes
}

\author{
Zeynep Gedik Özköse ${ }^{1}$ (D), Süleyman Cemil Oğlak² \\ ${ }^{1}$ Department of Perinatology, Kanuni Sultan Siileyman Training and Research Hospital, Health Sciences University, Istanbul, Turkey \\ ${ }^{2}$ Department of Obstetrics and Gynecology, Gazi Yaşargil Training and Research Hospital, Health Sciences University, Diyarbakur, Turkey
}

\begin{abstract}
Objective: This study aimed to determine the effect of advanced maternal age (AMA) on maternal and neonatal outcomes in pregnant women aged $\geq 35$ years compared with patients aged $30-34$ years. Also, we aimed to analyze the risk estimates of potential confounders to identify whether these variables contributed to the development of adverse pregnancy outcomes or not.

Methods: This retrospective cohort study included 2284 pregnant women aged $\geq 35$ years at the time of delivery who was delivered in a tertiary referral hospital from January 1, 2016, to December 31, 2020. We further classified these women into two subgroups: 35-39 years as early AMA (EAMA), and $\geq 40$ years as very AMA (VAMA). Pregnancy complications and adverse neonatal outcomes were recorded.

Results: Compared to younger women, pregnant AMA women had significantly higher risks of complicated pregnancies, including a higher risk of gestational diabetes mellitus (GDM, $\mathrm{p}<0.001$ ), polyhydramnios $(\mathrm{p}<0.001)$, cesarean section $(\mathrm{p}<0.001)$, stillbirths $(\mathrm{p}<0.001)$, major fetal abnormality $(\mathrm{p}<0.001)$, preterm delivery $(\mathrm{p}<0.001)$, lower birth weight $(\mathrm{p}<0.001)$, lower 5 -minute Apgar scores $(\mathrm{p}<0.001)$, lower umbilical artery blood $\mathrm{pH}$ values $(\mathrm{p}<0.001)$, neonatal intensive care unit (NICU) admission ( $\mathrm{p}<0.001)$, and length of NICU stay $(\mathrm{p}<0.001)$.

Conclusion: We found a strong and significant association between VAMA and adverse pregnancy outcomes, including an increased risk of GDM, polyhydramnios, cesarean section, and adverse neonatal outcomes, including a higher risk of stillbirths, preterm delivery, lower birth weight, lower 5-minute Apgar scores, and NICU admission.
\end{abstract}

Keywords: Advanced maternal age, pregnancy complications, adverse neonatal outcomes.

\section{Introduction}

The frequency of childbearing to women of advanced maternal age (AMA) had increased worldwide, particularly in the developed countries during the last decade. ${ }^{[1,2]}$ As reported by the Turkish Statistical Institute (TSI)
Özet: İleri maternal yaşın gebelik komplikasyonları ve advers neonatal sonuçlar üzerindeki etkileri

Amaç: Çalışmamızda, ileri maternal yaşın (AMA) 30-34 yaş arasındaki hastalara kıyasla $\geq 35$ yaşındaki gebelerde maternal ve neonatal sonuçlar üzerindeki etkisini belirlemeyi amaçladık. Ayrıca, karıştırıcı değişkenlerin advers gebelik sonuçlarının gelişimine katkıda bulunup bulunmadığını tespit etmek amacıyla potansiyel karıştırıcıların risk tahminlerini analiz etmeyi amaçladık.

Yöntem: Bu retrospektif kohort çalışması, doğum zamanında $\geq 35$ yaşında olan ve 1 Ocak 2016 ile 31 Aralık 2020 tarihleri arasında üçüncü basamak bir referans hastanesinde doğum yapan 2284 gebeyi içermektedir. Bu gebeleri iki alt gruba ayrıldı: 35-39 yaş arasında olanlar için erken AMA (EAMA) ve $\geq 40$ yaşında olanlar için geç AMA (VAMA). Gebelik komplikasyonları ve advers neonatal sonuçlar kaydedildi.

Bulgular: Daha genç kadınlara kıyasla AMA gebelerde; gestasyonel diabetes mellitus (GDM, $\mathrm{p}<0.001)$, polihidramniyos $(\mathrm{p}<0.001)$, sezaryen $(\mathrm{p}<0.001)$, ölü doğumlar $(\mathrm{p}<0.001)$, majör fetal anomali $(\mathrm{p}<0.001)$, preterm doğum $(\mathrm{p}<0.001)$, düşük doğum ağırlığı $(\mathrm{p}<0.001)$, düşük 5 . dakika Apgar skorları ( $\mathrm{p}<0.001)$, düşük umbilikal arter kanı $\mathrm{pH}$ değerleri $(\mathrm{p}<0.001)$, yenidoğan yoğun bakım ünitesine (YYBÜ) yatış $(\mathrm{p}<0.001)$ ve YYBÜ'de yatış uzunluğu $(\mathrm{p}<0.001)$ için daha yüksek risk dahil anlamlı şekilde daha yüksek komplike gebelik riski mevcuttu.

Sonuç: VAMA ile GDM, polihidramniyos ve sezaryen için artmış risk dahil advers gebelik sonuçları ve ölü doğum, preterm doğum, düşük doğum ağırlığı, düşük 5. dakika Apgar skoru ve YYBÜ'ye yatış için daha yüksek risk dahil advers neonatal sonuçlar arasında güçlü ve anlamlı bir ilişki tespit ettik.

Anahtar sözcükler: İleri maternal yaş, gebelik komplikasyonları, advers neonatal sonuçlar.

data in 2020, the age-specific birth rate among women aged $\geq 35$ years in Turkey was $0.60 \%$, whereas it was $0.52 \%$ in $2005 .^{[3]}$ The trend toward postponing pregnancy is primarily driven by social and cultural factors, extended education years, prioritization of career, later

Correspondence: Süleyman Cemil Oğlak, MD. Department of Obstetrics and Gynecology, Gazi Yaşargil Training and Research Hospital, Health Sciences University, Diyarbakır, Turkey. e-mail: sampson_21@hotmail.com / Received: October 12, 2021; Accepted: November 4, 2021

How to cite this article: Gedik Özköse Z, Oğlak SC. The implications for advanced maternal age on pregnancy complications and adverse neonatal outcomes. Perinatal Journal 2021;29(3):200-209. doi:10.2399/prn.21.0293005 
marriage, the implementation of effective contraceptive methods, developments in assisted reproductive technologies (ART), improvements in managing pre-existing chronic diseases, and easy access to these developments by patients. ${ }^{[4,5]}$

Previous studies reported that AMA has been considered as a risk factor for chromosomal abnormalities of the fetus and complications in the early stages of pregnancy, including ectopic pregnancy and miscarriage. ${ }^{[6]}$ However, studies on the associations between AMA and pregnancy outcomes have reported conflicting findings. Some studies have indicated that AMA has been associated with an increased risk of adverse maternal and neonatal outcomes, including preterm deliveries and related complications, gestational hypertensive disorders, gestational diabetes mellitus (GDM), placental abnormalities, stillbirths, and a higher frequency of cesarean section. ${ }^{[7,8]}$ But some investigations found no significant difference regarding pregnancy complications between older and younger pregnant women. ${ }^{[9,10]}$ Furthermore, the extent to which specified findings are associated with AMA and the strength of the relationship remained contradictory based on inadequately powered of the studies to adjust for potential confounding factors. ${ }^{[11]}$

This study aimed to determine the effect of AMA on maternal and neonatal outcomes in pregnant women aged $\geq 35$ years compared with patients aged $30-34$ years in a tertiary referral hospital. Also, we aimed to analyze the risk estimates of potential confounders to identify whether these variables contributed to the development of adverse pregnancy outcomes or not.

\section{Methods}

We conducted this retrospective cohort study in Kanuni Sultan Süleyman Training and Research Hospital, which was a tertiary referral hospital in Turkey, from January 1, 2016, to December 31, 2020. The ethics committee of the hospital approved the study (2021.01.6). We included all pregnant women aged $\geq 40$ years at the time of delivery and gave birth at 24 weeks of gestation or beyond during the study period. Also, a total of 2002 pregnant women aged between 35-39 years at the delivery time and who gave birth at or beyond 24 weeks' gestation were randomly selected by a computer system and were included in the study for further evaluation. Pregnant women lower than 30 years old, pregnancy loss $<24$ weeks of gestation, and pregnancies with missing medical records were excluded.
AMA was defined as pregnant women being 35 years or older at the time of delivery. We further classified these women into two subgroups: 35-39 years as early AMA (EAMA), and $\geq 40$ years as very AMA (VAMA). The study groups were compared with a control group which consisted of pregnant women aged between 30-34 years who gave birth at the same period. The control group patients were randomly selected by a computer system. All pregnant women included in this study were delivered and received postnatal care at our hospital, and if necessary, neonates were transferred to the neonatal intensive care unit (NICU). Also, the study subjects were categorized into two groups based on their parity as nulliparous and multiparous. A pregnant woman who has never given birth after 20 weeks of gestation or a neonate weighing more than $500 \mathrm{~g}$ was defined as nulliparous, and the remaining were defined as multiparous.

Maternal and neonatal data were collected from the hospital records to obtain information on maternal demographic characteristics, pregnancy complications, and obstetric and neonatal outcomes. Demographic characteristics included maternal age, body mass index (BMI), parity, the use of ART, and pre-existing at least one maternal chronic disease, including diabetes mellitus, chronic hypertension, hypothyroidism, bronchial asthma, cardiac diseases, known malignancy, and epilepsy. The occurrence of at least one adverse pregnancy outcome included gestational diabetes mellitus, preeclampsia, gestational hypertension, eclampsia, HELLP, premature rupture of membranes (PROM), small for gestational age (SGA) or intrauterine growth restriction (IUGR), placenta previa, polyhydramnios, oligohydramnios, and intrahepatic cholestasis of pregnancy (ICP). Obstetric outcomes included delivery mode (vaginal, or cesarean), and the need for postpartum blood transfusion due to postpartum hemorrhage. Neonatal outcomes included birth week, birth weight, major congenital abnormalities, stillbirth, 5-minute Apgar scores, NICU admission, and NICU length of stay.

The week of gestation was determined by ultrasonography (US) and was confirmed according to the firsttrimester US examination and last menstrual period. Gestational hypertensive disorders were diagnosed based on the ACOG criteria. ${ }^{[12]}$ We diagnosed GDM according to the 2010 International Association of Diabetes in Pregnancy Study Groups (IDPSG) criteria. ${ }^{[13]}$ Polyhydramnios was defined as an amniotic fluid index (AFI) $\geq 25$ utilizing the four-quadrant method, or by a single deepest pocket $>10 \mathrm{~cm}$ upon US examination. Oligohydramnios was defined as an $\mathrm{AFI}<5 \mathrm{~cm}$ in the US. 
We defined preterm birth as birth before 34 weeks of pregnancy. PPROM was described as a rupture of the fetal membranes before 37 weeks of completed gestation. ${ }^{[14]}$ ICP was diagnosed by the association of pruritus and bile acids $\geq 10 \mu \mathrm{mol} / \mathrm{L}$ (after other reasons of liver dysfunction and itching were ruled out) and by normalization of the levels of serum bile acids after birth. ${ }^{[15]}$ Placenta previa was described as the condition where the abnormal implantation of placental tissue overlying the endocervical os. We described the postpartum hemorrhage as the total blood loss of $\geq 1000 \mathrm{ml}$ within 24 hours after the delivery course (includes intrapartum loss) regardless of delivery mode. ${ }^{[16]}$ Birth weight was categorized into four categories as very low birth weight $(<1500$ g), low birth weight $(<2500 \mathrm{~g})$, normal birth weight (2500-4500 g), and high birth weight (macrosomia, $>4000 \mathrm{~g}$ ). SGA was defined as a weight below the 10th percentile for the gestational week. ${ }^{[17]}$ IUGR was defined as estimated fetal weight $<3$ rd percentile based on sonographic measurements of fetal biparietal diameter, head circumference, $\mathrm{AC}$, and femur length and no end-diastolic flow loss on Doppler examination. ${ }^{[18,19]}$ We defined stillbirth as a baby delivered with no signs of life known to have died after 24 completed weeks of pregnancy.

\section{Statistical analysis}

Numerical data were summarized as mean \pm standard deviation along with median (minimum-maximum), whereas frequency and percentage were used for categorical data. One-way ANOVA followed by Tukey's test for multiple comparisons was used to compare groups regarding a numerical variable. Chi-square test followed by Bonferroni corrected $\mathrm{z}$ test for multiple comparisons was used to compare groups regarding a categorical variable. Fisher's exact test was used for categorical variables in sparse contingency tables. Multiple logistic regression was performed to assess the risk of maternal age groups on neonatal outcomes and cesarean delivery considering potential confounders (i.e. ART pregnancies, parity, major fetal abnormality, multiple pregnancies). The results are presented showing odds ratios (OR) and 95\% confidence interval $(\mathrm{CI})$ estimates. All statistical tests were two-sided. A p-value $<0.05$ was considered statistically significant. Analyses were performed with $\mathrm{R}$ version 3.6.3 statistical computing language.

\section{Results}

During the study period, a total of 56,222 deliveries occurred, of which 282 women aged $\geq 40$ years were potentially suitable for including in the study. Also, a total of 2002 pregnant women aged between 35-39 years at the delivery time were constituted the EAMA group, and 2000 pregnant women aged between 30-34 years at the time of delivery were included in the control group. Patients in the EAMA and control groups were randomly selected by a computer system.

Table 1 demonstrates the demographic characteristics and clinical outcomes of the study subjects. The mean age of the VAMA group was $41.6 \pm 2.18$ years, the mean age of the EAMA group was $36.67 \pm 1.41$ years, and the mean age of the control group was $31.9 \pm 1.42$ years at the time of delivery. The nulliparous women were more prevalent in the age group $\geq 40$ years compared to the other two age groups. VAMA group $(9.2 \%)$ were more likely to have than the EAMA group $(2.9 \%)$ and the control group (3.1\%) to have conceived following ART $(\mathrm{p}<0.001)$, and the frequency of multiple pregnancies was recorded to be highest in the VAMA group $(\mathrm{p}<0.001)$, regardless of parity. No differences were observed among different age categories regarding BMI. The presence of at least one medical condition (diabetes mellitus, chronic hypertension, hypothyroidism, bronchial asthma, cardiac diseases, known malignancy, and epilepsy) was significantly higher in both of the AMA groups than in the younger mothers, regardless of parity $(p<0.001)$. Subgroup analysis of women aged $\geq 40$ years compared to women 35-39 years revealed that the presence of at least one medical condition was significantly higher in those over age 40 years $(\mathrm{p}<0.001)$. However, when we analyzed these medical conditions separately, we observed no significant differences across maternal age subgroups (Table 2). The occurrence of at least one adverse pregnancy outcome (GDM, preeclampsia, gestational hypertension, eclampsia, HELLP, premature rupture of membranes, SGA or IUGR, placenta previa, polyhydramnios, oligohydramnios, and ICP) was significantly higher in both of the AMA groups than in the younger mothers, regardless of parity $(\mathrm{p}<0.001)$. When we analyzed these adverse pregnancy outcomes separately, no significant differences were detected in all maternal age subgroups regarding preeclampsia, gestational hypertension, eclampsia, HELLP, premature rupture of membranes, SGA or IUGR, placenta previa, and oligohydramnios. As compared with the controls, pregnant AMA women were noted to have higher rates of GDM. Subgroup analysis revealed that the rates of GDM were significantly higher in the women aged $\geq 40$ years than those $35-39$ years $(p<0.001)$. The risk of polyhydramnios 
Table 1. The demographic characteristics and clinical outcomes of the study subjects.

\begin{tabular}{|c|c|c|c|c|}
\hline & \multicolumn{3}{|c|}{ Age groups } & \multirow[b]{2}{*}{ p-value } \\
\hline & $30-34$ & $35-39$ & 240 & \\
\hline Maternal age (years) & $\begin{array}{l}31.9 \pm 1.42 \\
32(30-34)\end{array}$ & $\begin{array}{c}36.67 \pm 1.41 * \\
37(35-39)\end{array}$ & $\begin{array}{c}41.6 \pm 2.18^{*,+} \\
37(35-39)\end{array}$ & $<0.001^{\ddagger}$ \\
\hline Nulliparous & $\begin{array}{l}31.78 \pm 1.41 \\
32(30-34)\end{array}$ & $\begin{array}{l}36.77 \pm 1.45^{*} \\
37(35-39)\end{array}$ & $\begin{array}{c}41.73 \pm 2.25^{*, \dagger} \\
41(40-49)\end{array}$ & $<0.001^{\ddagger}$ \\
\hline Multiparous & $\begin{array}{l}31.92 \pm 1.42 \\
32(30-34)\end{array}$ & $\begin{array}{l}36.75 \pm 1.40 * \\
37(35-39)\end{array}$ & $\begin{array}{c}41.53 \pm 2.17^{*}, \dagger \\
41(40-55)\end{array}$ & $<0.001^{\ddagger}$ \\
\hline \multicolumn{5}{|l|}{ Parity } \\
\hline Nulliparous & $311(15.6 \%)$ & $227(11.3 \%)$ & $51(18.1 \%)$ & \multirow{2}{*}{$<0.001 \S$} \\
\hline Multiparous & $1689(84.5 \%)$ & $1775(88.7 \%)$ & $231(81.9 \%)^{*}$ & \\
\hline Multiple pregnancies & $33(1.6 \%)$ & $87(4.3 \%)^{*}$ & $17(6 \%)^{*}$ & $<0.001 \S$ \\
\hline Nulliparous & $14(4.5 \%)$ & $23(10.1 \%)^{*}$ & $8(15.7 \%)^{*}$ & $0.004 \S$ \\
\hline Multiparous & $19(1.1 \%)$ & $64(3.6 \%)^{*}$ & $9(3.9 \%)^{*}$ & $<0.001 \S$ \\
\hline ART pregnancies & $63(3.1 \%)$ & $58(2.9 \%)$ & $26(9.2 \%)^{*, \dagger}$ & $<0.001^{\S}$ \\
\hline Nulliparous & $53(17 \%)$ & $37(16.3 \%)$ & $17(33.3 \%)^{*, \dagger}$ & $0.013^{\S}$ \\
\hline Multiparous & $10(0.6 \%)$ & $21(1.2 \%)$ & $9(3.9 \%)^{*, \dagger}$ & $<0.001 \S$ \\
\hline \multicolumn{5}{|l|}{ BMI $\left(\mathrm{kg} / \mathrm{m}^{2}\right)$} \\
\hline$<25$ & $563(28.1 \%)$ & $563(28.1 \%)$ & $76(27 \%)$ & \multirow{3}{*}{$0.985 \S$} \\
\hline $25-30$ & $412(20.6 \%)$ & $413(20.6 \%)$ & $62(22 \%)$ & \\
\hline$\geq 30$ & $1026(51.3 \%)$ & $1026(51.2 \%)$ & $144(51.1 \%)$ & \\
\hline BMI (mean \pm standard deviation) & $\begin{array}{l}29.91 \pm 7.71 \\
30(16-49)\end{array}$ & $\begin{array}{l}29.91 \pm 7.71 \\
30(16-49)\end{array}$ & $\begin{array}{l}29.45 \pm 6.98 \\
30(16-48)\end{array}$ & $0.658^{\ddagger}$ \\
\hline Nulliparous & $\begin{array}{l}29.05 \pm 7.52 \\
29(16-49)\end{array}$ & $\begin{array}{l}30.46 \pm 8.01 \\
30(16-46)\end{array}$ & $\begin{array}{l}30.67 \pm 7.04 \\
31(18-48)\end{array}$ & $0.071^{\ddagger}$ \\
\hline Multiparous & $\begin{array}{l}30.08 \pm 7.73 \\
30(16-49)\end{array}$ & $\begin{array}{l}29.84 \pm 7.66 \\
30(16-49)\end{array}$ & $\begin{array}{l}29.22 \pm 6.95 \\
29(17-46) \\
\end{array}$ & $0.242^{\ddagger}$ \\
\hline At least one additional medical condition & $68(3.4 \%)$ & $148(7.4 \%)^{*}$ & $75(26.6 \%)^{*, \dagger}$ & $<0.001 \S$ \\
\hline Nulliparous & $15(4.8 \%)$ & $33(14.5 \%)^{*}$ & $13(25.5 \%)^{*}$ & $<0.001 \S$ \\
\hline Multiparous & $53(3.1 \%)$ & $115(6.5 \%)^{*}$ & $62(26.6 \%)^{*, \dagger}$ & $<0.001^{\S}$ \\
\hline At least one adverse pregnancy outcome & $509(25.5 \%)$ & $627(31.3 \%)^{*}$ & $199(70.6 \%)^{*, \dagger}$ & $<0.001^{\S}$ \\
\hline Nulliparous & $105(33.8 \%)$ & $96(42.3 \%)^{*}$ & $34(66.7 \%)^{*,+}$ & $<0.001 \S$ \\
\hline Multiparous & $404(23.9 \%)$ & $531(29.9 \%)^{*}$ & $165(71.4 \%)^{*, \dagger}$ & $<0.001 \S$ \\
\hline Type of delivery (cesarean section) & $1065(53.2 \%)$ & $1318(65.8 \%)^{*}$ & $217(77 \%)^{*},+$ & $<0.001 \S$ \\
\hline Nulliparous & $169(54.3 \%)$ & $154(67.8 \%)^{*}$ & $44(86.3 \%)^{*, \dagger}$ & $<0.001^{\S}$ \\
\hline Multiparous & $896(53 \%)$ & $1164(65.6 \%)^{*}$ & $173(74.9 \%)^{*, \dagger}$ & $<0.001^{\S}$ \\
\hline Postpartum blood transfusion & $90(4.5 \%)$ & $92(4.6 \%)$ & $15(5.3 \%)$ & $0.827 \S$ \\
\hline Nulliparous & $10(3.2 \%)$ & $14(6.2 \%)$ & $1(2 \%)$ & $0.171 \S$ \\
\hline Multiparous & $80(4.7 \%)$ & $78(4.4 \%)$ & $14(6.1 \%)$ & $0.515 \S$ \\
\hline
\end{tabular}

Numerical data were summarized as mean \pm standard deviation along with median (minimum-maximum), whereas frequency and percentage were used for categorical data. *Significantly different than age group 30-34 (control); †Significantly different than age group 35-39, according to multiple comparisons. p-values are based on $\neq_{0}$ ene way ANOVA, §chi-square test.

was particularly higher in multiparous women aged $\geq 40$ years than the EAMA women and younger controls $(\mathrm{p}=0.002)$. However, in nulliparous pregnancies, the occurrence of polyhydramnios was not significantly different in the three groups.

The rates of cesarean delivery were increased in each of the AMA subgroups relative to the younger controls, regardless of parity $(\mathrm{p}<0.001)$. Subgroup analysis of women aged $35-39$ years compared to women $\geq 40$ years revealed that the cesarean delivery rate was significantly higher in those over age 40 years. All age groups were comparable regarding the incidence of postpartum blood transfusion due to postpartum hemorrhage, regardless of parity.

Table 3 presents the neonatal outcomes of the older and younger mothers. There was a statistically signifi- 
Table 2. Additional medical conditions and adverse pregnancy outcomes of the study subjects.

\begin{tabular}{|c|c|c|c|c|}
\hline & \multicolumn{3}{|c|}{ Age groups } & \multirow[b]{2}{*}{ p-value } \\
\hline & 30-34 & $35-39$ & $\geq 40$ & \\
\hline \multicolumn{5}{|l|}{ Additional medical conditions } \\
\hline Diabetes mellitus & $30(42.3 \%)$ & $35(28 \%)$ & $29(32 \%)$ & $0.127 \S$ \\
\hline Nulliparous & $6(37.5 \%)$ & $9(30 \%)$ & $4(25 \%)$ & $0.741^{\S}$ \\
\hline Multiparous & $24(43.6 \%)$ & $26(27.7 \%)$ & $25(33.3 \%)$ & $0.135^{ \pm}$ \\
\hline Chronic hypertension & $24(33.8 \%)$ & $40(32 \%)$ & $40(44.4 \%)$ & $0.185 \S$ \\
\hline Nulliparous & $4(25 \%)$ & $8(26.7 \%)$ & $8(50 \%)$ & $1.258 \S$ \\
\hline Multiparous & $20(36.4 \%)$ & $32(34 \%)$ & $32(42.7 \%)$ & $0.524 \S$ \\
\hline Hypothyroidism & $7(9.9 \%)$ & $21(16.8 \%)$ & $15(16.7 \%)$ & $0.370 \S$ \\
\hline Nulliparous & $3(18.8 \%)$ & $6(20 \%)$ & $2(12.5 \%)$ & $0.912 \S$ \\
\hline Multiparous & $4(7.3 \%)$ & $15(16 \%)$ & $13(17.3 \%)$ & $0.077^{\ddagger}$ \\
\hline Bronchial asthma & $3(4.2 \%)$ & $8(6.4 \%)$ & $4(4.4 \%)$ & $0.561^{\ddagger}$ \\
\hline Nulliparous & $1(6.3 \%)$ & $1(3.3 \%)$ & $0(0 \%)$ & $0.317^{\ddagger}$ \\
\hline Multipar & $2(3.6 \%)$ & $7(7.4 \%)$ & $4(5.3 \%)$ & $0.452^{\ddagger}$ \\
\hline Cardiac disease & $4(5.6 \%)$ & $12(9.6 \%)$ & $3(3.4 \%)$ & $0.281^{\neq}$ \\
\hline Nulliparous & $1(6.3 \%)$ & $3(10 \%)$ & $2(12.5 \%)$ & $0.385^{\ddagger}$ \\
\hline Multiparous & $3(5.5 \%)$ & $9(9.6 \%)$ & $1(1.3 \%)$ & $0.159^{\ddagger}$ \\
\hline Known malignancy & $1(1.4 \%)$ & $4(3.2 \%)$ & $0(0 \%)$ & $0.305^{ \pm}$ \\
\hline Nulliparous & $0(0 \%)$ & $0(0 \%)$ & $0(0 \%)$ & NA \\
\hline Multiparous & $1(1.8 \%)$ & $4(4.3 \%)$ & $0(0 \%)$ & $0.286^{\ddagger}$ \\
\hline Epilepsy & $2(2.8 \%)$ & $4(3.2 \%)$ & $0(0 \%)$ & $0.147^{\ddagger}$ \\
\hline Nulliparous & $1(6.3 \%)$ & $3(10 \%)$ & $0(0 \%)$ & $0.363^{\ddagger}$ \\
\hline Multiparous & $1(1.8 \%)$ & $1(1.1 \%)$ & $0(0 \%)$ & $0.266 \S$ \\
\hline \multicolumn{5}{|l|}{ Adverse pregnancy outcomes } \\
\hline Gestational diabetes & $71(3.6 \%)$ & $125(6.2 \%)^{*}$ & $43(15.2 \%)^{*,+}$ & $<0.001^{\S}$ \\
\hline Nulliparous & $15(4.8 \%)$ & $15(6.6 \%)^{*}$ & $7(13.7 \%)^{*, \dagger}$ & $0.041^{\S}$ \\
\hline Multiparous & $56(3.3 \%)$ & $110(6.2 \%)^{*}$ & $36(15.6 \%)^{*,+}$ & $<0.001^{\S}$ \\
\hline Preeclampsia & $153(25.5 \%)$ & $192(28.1 \%)$ & $65(24.4 \%)$ & $0.421 \S$ \\
\hline Nulliparous & $32(24.8 \%)$ & $41(34.2 \%)$ & $12(25 \%)$ & $0.219 \S$ \\
\hline Multiparous & $121(25.7 \%)$ & $151(26.8 \%)$ & $53(24.3 \%)$ & $0.774 \S$ \\
\hline Gestational hypertension & $34(5.7 \%)$ & $38(5.6 \%)$ & $12(4.3 \%)$ & $0.697 \S$ \\
\hline Nulliparous & $6(4.7 \%)$ & $4(3.3 \%)$ & $1(2.1 \%)$ & $0.774^{\ddagger}$ \\
\hline Multiparous & $28(6 \%)$ & $34(6 \%)$ & $11(4.8 \%)$ & $0.789 \S$ \\
\hline HELLP & $1(0.2 \%)$ & $4(0.6 \%)$ & $0(0 \%)$ & $0.280^{\ddagger}$ \\
\hline Nulliparous & $1(0.8 \%)$ & $1(0.8 \%)$ & $0(0 \%)$ & $1.000^{\ddagger}$ \\
\hline Multiparous & $0(0 \%)$ & $3(0.5 \%)$ & $0(0 \%)$ & $0.224^{\ddagger}$ \\
\hline Eclampsia & $2(0.3 \%)$ & $0(0 \%)$ & $0(0 \%)$ & $0.179^{\ddagger}$ \\
\hline Nulliparous & $0(0 \%)$ & $0(0 \%)$ & $0(0 \%)$ & NA \\
\hline Multiparous & $2(0.4 \%)$ & $0(0 \%)$ & $0(0 \%)$ & $0.171^{\ddagger}$ \\
\hline Premature rupture of membranes & $93(15.5 \%)$ & $115(16.8 \%)$ & $32(12 \%)$ & $0.188^{\S}$ \\
\hline Nulliparous & $23(17.8 \%)$ & $23(19.2 \%)$ & $6(12.5 \%)$ & $0.585^{\S}$ \\
\hline Multiparous & $70(14.9 \%)$ & $92(16.3 \%)$ & $26(11.9 \%)$ & $0.304 \S$ \\
\hline SGA/IUGR & $109(18.2 \%)$ & $130(19 \%)$ & $38(14.8 \%)$ & $0.331 \S$ \\
\hline Nulliparous & $30(23.3 \%)$ & $18(15 \%)$ & $12(25 \%)$ & $0.179 \S$ \\
\hline Multiparous & $99(76.7 \%)$ & $102(85 \%)$ & $36(75 \%)$ & $0.051 \S$ \\
\hline Placenta previa & $82(13.9 \%)$ & $108(15.8 \%)$ & $42(15.8 \%)$ & $0.584 \S$ \\
\hline Nulliparous & $10(7.8 \%)$ & $4(3.3 \%)$ & $6(12.5 \%)$ & $0.095^{\|}$ \\
\hline Multiparous & $73(15.5 \%)$ & $104(18.4 \%)$ & $36(16.5 \%)$ & $0.453 \S$ \\
\hline Polyhydramnios & $6(1 \%)$ & $27(3.9 \%)$ & $14(5.3 \%)^{*,+}$ & $0.001 \S$ \\
\hline Nulliparous & $0(0 \%)$ & $1(0.8 \%)$ & $1(2.1 \%)$ & $0.309^{\ddagger}$ \\
\hline Multiparous & $6(1.3 \%)$ & $26(4.6 \%)$ & $13(6 \%)^{*, \dagger}$ & $0.002 \S$ \\
\hline Oligohydramnios & $25(4.2 \%)$ & $37(5.4 \%)$ & $7(2.6 \%)$ & $0.161 \S$ \\
\hline Nulliparous & $6(4.7 \%)$ & $4(3.3 \%)$ & $1(2.1 \%)$ & $0.774 \|$ \\
\hline Multiparous & $19(4 \%)$ & $33(5.9 \%)$ & $6(2.8 \%)$ & $0.135 \S$ \\
\hline Intrahepatic cholestasis & $22(3.7 \%)$ & $14(2 \%)$ & $3(1.1 \%)$ & $0.051^{\S}$ \\
\hline Nulliparous & $6(4.7 \%)$ & $5(4.2 \%)$ & $2(4.2 \%)$ & $1.000^{\ddagger}$ \\
\hline Multiparous & $16(3.4 \%)$ & $9(1.6 \%)$ & $1(0.5 \%)^{*, \dagger}$ & $0.022^{\ddagger}$ \\
\hline
\end{tabular}

Data were summarized as frequency and percentage. 'Significantly different than age group 35-39, according to multiple comparisons. p-values are based on §chi-square test; "Fisher's exact test. NA: not available, no statistics are computed because of insufficient data. 
Table 3. Neonatal outcomes of the patients.

\begin{tabular}{|c|c|c|c|c|}
\hline & \multicolumn{3}{|c|}{ Age groups } & \multirow[b]{2}{*}{ p-value } \\
\hline & $30-34$ & $35-39$ & 240 & \\
\hline Gestational age at birth & $\begin{array}{l}36.7 \pm 3.69 \\
38(17-42)\end{array}$ & $\begin{array}{l}35.9 \pm 4.53^{*} \\
38(19-42)\end{array}$ & $\begin{array}{c}33.15 \pm 4.40^{*, \dagger} \\
34(23-40)\end{array}$ & $<0.001^{\ddagger}$ \\
\hline Nulliparous & $\begin{array}{l}35.56 \pm 4.47 \\
37(20-41)\end{array}$ & $\begin{array}{c}34.03 \pm 5.52 * \\
36(24-42)\end{array}$ & $\begin{array}{c}32.9 \pm 4.57 \text { * } \\
34(24-40)\end{array}$ & $<0.001^{\ddagger}$ \\
\hline Multiparous & $\begin{array}{l}36.87 \pm 3.49 \\
38(17-42)\end{array}$ & $\begin{array}{c}36.12 \pm 4.33^{*} \\
38(19-42)\end{array}$ & $\begin{array}{c}33.2 \pm 4.37 *, \dagger \\
34(23-40)\end{array}$ & $<0.001^{\ddagger}$ \\
\hline Birth weight (gram) & $\begin{array}{c}2867.87 \pm 860.3 \\
3050(245-5040)\end{array}$ & $\begin{array}{c}2836.42 \pm 948.4 \\
3000(100-5640)\end{array}$ & $\begin{array}{l}2120.98 \pm 1000^{*,+} \\
2240(100-5000)\end{array}$ & $<0.001^{\ddagger}$ \\
\hline Nulliparous & $\begin{array}{c}2529 \pm 947.21 \\
2700(250-4720)\end{array}$ & $\begin{array}{l}2503.03 \pm 1090.08 \\
2890(200-5110) \\
\end{array}$ & $\begin{array}{c}2076.47 \pm 923.48^{*, \dagger}+ \\
2300(420-4330)\end{array}$ & $0.011^{\neq}$ \\
\hline Multiparous & $\begin{array}{l}2930.12 \pm 828.92 \\
3090(245-5040)\end{array}$ & $\begin{array}{l}2879.06 \pm 320.39 \\
3035(100-5640)\end{array}$ & $\begin{array}{c}2130.8 \pm 1017.85^{*}, \dagger \\
2205(100-5000)\end{array}$ & $<0.001^{\ddagger}$ \\
\hline Gender, male & $1029(51.6 \%)$ & $948(50.2 \%)$ & $132(47 \%)$ & $0.317 \S$ \\
\hline Nulliparous & $169(54.3 \%)$ & $89(44.9 \%)$ & $22(43.1 \%)$ & $0.070^{\S}$ \\
\hline Multiparous & $860(51.1 \%)$ & $859(50.9 \%)$ & $110(47.8 \%)$ & $0.650 \S$ \\
\hline Major fetal anomaly & $57(2.9 \%)$ & $67(3.4 \%)$ & $27(9.6 \%)^{*,+}$ & $<0.001 \S$ \\
\hline Nulliparous & $13(4.4 \%)$ & $6(2.6 \%)$ & $2(3.9 \%)$ & 0.578 \\
\hline Multiparous & $44(2.7 \%)$ & $61(3.5 \%)$ & $25(10.8 \%)^{*, \dagger}$ & $<0.001 \S$ \\
\hline Stillbirth & $72(3.6 \%)$ & $142(7.1 \%)^{*}$ & $30(10.6 \%)^{*}$ & $<0.001^{\S}$ \\
\hline Nulliparous & $17(5.5 \%)$ & $22(9.7 \%)$ & $6(7.6 \%)$ & $0.097 \S$ \\
\hline Multiparous & $55(3.3 \%)$ & $12(6.8 \%)^{*}$ & $24(10.4 \%)^{*}$ & $<0.001 \S$ \\
\hline Preterm birth $<34$ weeks & $305(15.3 \%)$ & $248(21.3 \%)^{*}$ & $118(41.8 \%)^{*, \dagger}$ & $<0.001 \S$ \\
\hline Nulliparous & $79(25.4 \%)$ & $48(34.8 \%)^{*}$ & $23(45.1 \%)^{*,+}$ & $0.006^{\S}$ \\
\hline Multiparous & $226(13.4 \%)$ & $200(19.5 \%)^{*}$ & $95(41.1 \%)^{*, \dagger}$ & $<0.001 \S$ \\
\hline \multicolumn{5}{|l|}{ Birth weight } \\
\hline$<2500 \mathrm{~g}$ & $544(27.2 \%)$ & $527(26.3 \%)$ & $169(59.9 \%)^{*, \dagger}$ & $<0.001 \S$ \\
\hline Nulliparous & $132(42.4 \%)$ & $91(40.1 \%)$ & $31(60.8 \%)^{*, \dagger}$ & $0.025 \S$ \\
\hline Multiparous & $412(24.4 \%)$ & $436(24.6 \%)$ & $138(59.7 \%)^{*,+}$ & $<0.001 \S$ \\
\hline$<1500 \mathrm{~g}$ & $200(10 \%)$ & $236(11.8 \%)$ & $84(29.8 \%)^{*,+}$ & $<0.001^{\S}$ \\
\hline Nulliparous & $53(17 \%)$ & $49(21.6 \%)$ & $12(23.5 \%)$ & $0.307 \S$ \\
\hline Multiparous & $147(8.7 \%)$ & $187(10.5 \%)$ & $72(31.2 \%)^{*,+}$ & $<0.001 \S$ \\
\hline$>4000 \mathrm{~g}$ & $107(5.3 \%)$ & $110(5.5 \%)$ & $6(2.1 \%)$ & $0.055 \S$ \\
\hline Nulliparous & $10(3.2 \%)$ & $10(4.4 \%)$ & $1(2 \%)$ & $0.628^{\S}$ \\
\hline Multiparous & $97(5.7 \%)$ & $100(5.6 \%)$ & $5(2.2 \%)$ & $0.073 \S$ \\
\hline 5-minute Apgar $<7$ & $171(8.6 \%)$ & $228(11.4 \%)^{*}$ & $50(17.7 \%)^{*,+}$ & $<0.001 \S$ \\
\hline Nulliparous & $43(13.8 \%)$ & $37(16.4 \%)$ & $8(15.7 \%)$ & $0.709 \S$ \\
\hline Multiparous & $128(7.6 \%)$ & $191(10.8 \%)^{*}$ & $42(18.2 \%)^{*,+}$ & $<0.001 \S$ \\
\hline Neonatal ICU & $316(15.8 \%)$ & $546(27.3 \%)^{*}$ & $107(37.9 \%)^{*,+}$ & $<0.001^{\S}$ \\
\hline Nulliparous & $52(16.7 \%)$ & $71(31.3 \%)^{*}$ & $22(43.1 \%)^{*,+}$ & $0.028 \S$ \\
\hline Multiparous & $264(15.6 \%)$ & $475(26.8 \%)^{*}$ & $85(36.8 \%)^{*,+}$ & $<0.001 \S$ \\
\hline Neonatal ICU (days) & $\begin{array}{l}13.66 \pm 10.1 \\
10(1-120)\end{array}$ & $\begin{array}{c}19.5 \pm 20.26^{*} \\
10(1-143)\end{array}$ & $\begin{array}{c}34.71 \pm 31.2^{*, \dagger} \\
27(1-190)\end{array}$ & $<0.001^{\ddagger}$ \\
\hline Nulliparous & $\begin{array}{l}15.59 \pm 11.65 \\
12(2-70)\end{array}$ & $\begin{array}{c}22.18 \pm 20.7^{*} \\
14(3-117)\end{array}$ & $\begin{array}{l}33.77 \pm 36.1^{*,+} \\
22.5(0-150)\end{array}$ & $0.001^{\ddagger}$ \\
\hline Multiparous & $\begin{array}{l}13.37 \pm 9.79 \\
10(1-120)\end{array}$ & $\begin{array}{c}18.63 \pm 20.06^{*} \\
10(1-143)\end{array}$ & $\begin{array}{c}34.95 \pm 31.07 *,+ \\
27(1-190)\end{array}$ & $<0.001^{\ddagger}$ \\
\hline Umbilical cord blood pH & $\begin{array}{c}7.29 \pm 0.06 \\
7.3(6.7-7.8)\end{array}$ & $\begin{array}{l}7.28 \pm 0.07^{*} \\
7.3(6.6-7.6)\end{array}$ & $\begin{array}{l}7.25 \pm 0.11^{*,+} \\
7.3(6.8-7.4)\end{array}$ & $<0.001^{\ddagger}$ \\
\hline Nulliparous & $\begin{array}{c}7.28 \pm 0.09 \\
7.3(6.8-7.8)\end{array}$ & $\begin{array}{l}7.28 \pm 0.07^{*} \\
7.3(6.8-7.3)\end{array}$ & $\begin{array}{l}7.24 \pm 0.13^{*,+} \\
7.3(6.8-7.3)\end{array}$ & $0.047^{\ddagger}$ \\
\hline Multiparous & $\begin{array}{c}7.29 \pm 0.05 \\
7.3(6.6-7.6)\end{array}$ & $\begin{array}{l}7.28 \pm 0.06^{*} \\
7.3(6.6-7.6)\end{array}$ & $\begin{array}{l}7.25 \pm 0.11^{*,+} \\
7.3(6.8-7.4)\end{array}$ & $<0.001^{\ddagger}$ \\
\hline
\end{tabular}

Numerical data were summarized as mean \pm standard deviation along with median (minimum-maximum), whereas frequency and percentage were used for categorical data. *Significantly different than age group 30-34 (control); †Significantly different than age group 35-39, according to multiple comparisons. p-values are based on ₹one way ANOVA, schi-square test. 
cant increase in the incidence of major fetal anomalies in multiparous women in the VAMA group $(\mathrm{p}<0.001)$, whereas no difference was found in the incidence of major fetal anomalies in nulliparous pregnancies as the maternal age increased. In multiparous pregnancies with AMA, there was a significantly increased incidence of stillbirth among neonates born to older women relative to the younger controls $(\mathrm{p}<0.001)$. However, the incidence of stillbirth was not associated with AMA in nulliparous pregnancies. When comparing multiparous AMA to younger women, the percent of preterm birth was significantly increased in all the AMA age subgroups $(\mathrm{p}<0.001)$. However, when comparing only nulliparous AMA to younger controls, the proportion of preterm birth was significantly increased only in the VAMA subgroup ( $\mathrm{p}=0.006)$. The percent of low birth weight was significantly increased among neonates born to VAMA women as compared to the neonates of EAMA and younger controls $(\mathrm{p}<0.001)$. The proportion of very low birth was significantly increased only in the neonates born to multiparous VAMA women as compared to the newborns of multiparous EAMA and multiparous women in the control group $(\mathrm{p}<0.001)$, while the percent of very low birth was not significantly different between nulliparous AMA women and younger nulliparous controls. The frequency of high birth weight in infants born to older women was not significantly different from the proportion seen in the women in the control group. There was a statistically significant increase in the incidence of 5-minute Apgar scores of $<7$ in mul- tiparous women in the EAMA and VAMA group in comparison to the control group $(\mathrm{p}<0.001)$, whereas no difference was observed in the incidence of 5-minute Apgar scores of $<7$ in nulliparous pregnancies as the maternal age increased. A significantly higher incidence of NICU admission and a significantly longer NICU length of stay was found among neonates born to the VAMA group relative to the EAMA group and younger controls, regardless of parity $(\mathrm{p}<0.001)$. There was a statistically significant decrease in the umbilical cord blood $\mathrm{pH}$ value in nulliparous and multiparous pregnancies in the VAMA group relative to the EAMA group and younger controls, regardless of parity $(\mathrm{p}<0.001)$.

We conducted a multiple logistic regression analysis to adjust the variables, including parity, ART pregnancies, multiple pregnancies, and major fetal abnormalities and summarized the in Table 4. We found that VAMA is a significant independent risk factor for the occurrence of adverse pregnancy outcomes, including GDM $(\mathrm{OR}=4.95$, $95 \% \mathrm{CI}=3.30-7.42, \mathrm{p}<0.001)$, polyhydramnios $(\mathrm{OR}=5.92$, 95\% CI $=2.92-11.98, \mathrm{p}<0.001)$, and cesarean section $(\mathrm{OR}=2.74,95 \% \mathrm{CI}=2.04-3.67, \mathrm{p}<0.001)$, and adverse neonatal outcomes, including stillbirth $(\mathrm{OR}=2.55,95 \%$ $\mathrm{CI}=1.57-4.13, \mathrm{p}<0.001)$, preterm birth $(\mathrm{OR}=3.54,95 \%$ $\mathrm{CI}=2.68-4.67, \mathrm{p}<0.001)$, low birth weight $(\mathrm{OR}=3.58$, 95\% CI=2.74-4.68, $\mathrm{p}<0.001), 5$-minute Apgar scores of $<7(\mathrm{OR}=1.80,95 \% \mathrm{CI}=1.24-2.61, \mathrm{p}=0.002)$, and the need for NICU admission (OR=0.70, 95\% CI $=0.53-0.91$, $\mathrm{p}=0.008)$.

Table 4. Multiple logistic regression results of the adverse pregnancy outcomes and neonatal outcomes.

\begin{tabular}{|c|c|c|c|c|}
\hline & \multicolumn{4}{|c|}{ Age groups } \\
\hline & \multicolumn{2}{|c|}{ 35-39 } & \multicolumn{2}{|c|}{$\geq 40$} \\
\hline & Adj. OR (95\% Cl) & p-value & Adj. OR (95\% Cl) & p-value \\
\hline Stillbirth & $2.18(1.60-2.96)^{*}$ & $<0.001$ & $2.55(1.57-4.13)^{*}$ & $<0.001$ \\
\hline Preterm birth & $1.52(1.25-1.84)^{*}$ & $<0.001$ & $3.54(2.68-4.67)^{*}$ & $<0.001$ \\
\hline Birth weight $<2500 \mathrm{~g}$ & $0.92(0.79-1.06)^{*}$ & 0.240 & $3.58(2.74-4.68)^{*}$ & $<0.001$ \\
\hline Birth weight $<1500 \mathrm{~g}$ & $1.20(0.97-1.47)^{\star}$ & 0.089 & $3.26(2.40-4.43)^{*}$ & $<0.001$ \\
\hline 5-minute Apgar $<7$ & $1.37(1.10-1.71)^{\star}$ & 0.005 & $1.80(1.24-2.61)^{\star}$ & 0.002 \\
\hline Neonatal ICU & $1.01(0.88-1.17)^{*}$ & 0.889 & $1.44(1.10-1.88)^{*}$ & 0.008 \\
\hline Type of delivery (cesarean section) & $1.66(1.46-1.88)^{\dagger}$ & $<0.001$ & $2.74(2.04-3.67)^{\dagger}$ & $<0.001$ \\
\hline Gestational diabetes & $1.85(1.37-2.50)^{\dagger}$ & $<0.001$ & $4.95(3.30-7.42)^{\dagger}$ & $<0.001$ \\
\hline Polyhydramnios & $1.39(0.77-2.52)^{+}$ & 0.271 & $5.92(2.92-11.98)^{\dagger}$ & $<0.001$ \\
\hline
\end{tabular}

Adj.: Adjusted for *ART pregnancies, parity, major fetal anomaly, multiple pregnancies; ${ }^{\dagger}$ ART pregnancies, parity, multiple pregnancies. Reference group is women aged 30-34. 


\section{Discussion}

The current study demonstrates that compared to younger women, pregnant women aged $>35$ years have significantly higher risks of complicated pregnancies, including a higher risk of GDM, polyhydramnios, cesarean section, stillbirths, major fetal abnormality, preterm delivery, lower birth weight, lower 5-minute Apgar scores, lower umbilical artery blood $\mathrm{pH}$-values, NICU admission, and length of NICU stay. Also, we performed a multiple logistic regression analysis to adjust the variables, including parity, ART pregnancies, multiple pregnancies, and major fetal abnormalities, and found that VAMA is significantly associated with adverse pregnancy outcomes, including a higher risk of gestational diabetes mellitus, polyhydramnios, cesarean section, and adverse neonatal outcomes, including an increased risk of stillbirths, preterm delivery, lower birth weight, lower 5minute Apgar scores, and NICU admission.

Many studies have reported a higher incidence of gestational hypertensive disorders among pregnant women with AMA. ${ }^{[20-22]}$ The higher risk of developing early-onset preeclampsia may be explained by the ageassociated vascular endothelial damage and dysfunction, which is incapable of handling cope with the substantial physiological cardiovascular changes of pregnancy. ${ }^{[23]}$ Contrary to the literature, Cakmak Celik et al.'s study from Turkey reported no significant difference in the preeclampsia incidence in AMA women, in agreement with our study. ${ }^{[24]}$ In Turkey, pregnant women with gestational hypertensive diseases are frequently referred to and managed in tertiary care centers. We considered that the tendency of referring the patients with a diagnosis of preeclampsia to the tertiary centers leads to the similarity of preeclampsia incidence in younger and older patients in Turkey.

Previous studies found a significant correlation between AMA and GDM, in line with our research. ${ }^{[2,25,26]}$ This correlation might be due to the potential impairment of carbohydrate metabolism that occurs with increasing age. ${ }^{[2]}$ Also, we consider that the higher frequency of GDM in AMA women was not associated with maternal adipose tissue since we detected no significant differences between the groups regarding the maternal BMI. Also, we analyzed patients with pre-existing diabetes mellitus separately and excluded them from the subgroup of women diagnosed with GDM. We found similar rates of pre-existing diabetes between the groups. As a consequence of the higher rates of GDM in AMA women, we found a significantly higher incidence of polyhydramnios in the EAMA and VAMA groups compared to the control group.

Numerous studies identified a higher incidence of cesarean delivery among pregnant women aged $>35$ years and reported that AMA is a strong risk factor for cesarean delivery. ${ }^{[5,20,22,25-27]}$ Kanmaz et al. stated that pregnancy complications being more common in AMA pregnancies, increased uterine surgery rates, higher rates of ART pregnancies and increased non-vertex fetal presentation rates have a significant role in this result. ${ }^{[26]}$ Ates et al. indicated that older nulliparous women usually have an extended story of infertility and the possibility of this being the only pregnancy might affect the clinician's choices regarding the delivery route. ${ }^{[20]}$ Also, higher cesarean section rates might reflect a tendency for physicians to recommend cesarean delivery in VAMA women. ${ }^{[2,27]}$ In our clinic, we determined the delivery route by standard obstetric indications except for the ART pregnancies. We attributed the higher rates of cesarean birth in AMA women to complicated pregnancies being more common in AMA pregnancies and higher rates of ART pregnancies.

Saleh Gargari et al. reported that the most crucial risk factor for the occurrence of placenta previa was AMA. ${ }^{[28]}$ Contrary to Saleh Gargari et al., we found similar rates of placenta previa in all age groups since pregnancies with placenta previa were frequently referred to a tertiary center in all age groups in our country. Yogev et al. found a significant correlation between AMA and postpartum hemorrhage and the need for blood transfusion. ${ }^{[2]}$ However, Lao et al. indicated that aging was related to decreased postpartum hemorrhage, the risk decreasing progressively from those aged 25-29 years to those aged $\geq 40$ years. ${ }^{[30]} \mathrm{We}$ did not detect a higher risk of postpartum hemorrhage in EAMA and VAMA groups.

Maternal age of more than 35 years is a crucial risk factor that is related to a $65 \%$ increase in the odds of stillbirth. $^{[31]}$ The physiopathological mechanism of the increase in stillbirth risk with AMA is unclear. Low uteroplacental perfusion caused by poor uterine vasculature in older women has been suggested the direct effect of maternal aging. The increased risk might also be attributed to the correlation between AMA and definite risk factors for stillbirths, including obstetric and medical complications. ${ }^{[2]}$ Likewise, we found a significant ageassociated increase in the stillbirth incidence in particular.

Women at AMA were at higher risk of delivery before 34 weeks of pregnancy and had low birth weight. ${ }^{[11,22,25,27]}$ Frederiksen et al. attributed this to 
obstetric or medical complications reported to increase with older maternal ages. ${ }^{[1]}$ Marozio et al. noted that the higher frequency of preterm delivery and low birth weight was primarily due to iatrogenic preterm delivery indicated for obstetric complications. ${ }^{[2]}$ Fitzpatrick et al. found that the rates of both spontaneous and iatrogenic preterm birth were higher in women of AMA than in the younger mothers. ${ }^{[27]} \mathrm{We}$ also demonstrated a significant association between AMA and higher risk of preterm delivery and low birth weight without separating the type of preterm delivery. In our study population, the rates of fetuses with SGA and IUGR were similar between the groups. Thus, we consider that the higher rate of low birth weight infants was predominantly linked to the high preterm birth rate. As expected, we show that a 5 -minute Apgar score $<7$, which is significantly associated with long-term cognitive outcomes, and NICU admission were more frequent and length of NICU admission stay was longer among neonates of AMA women in comparison to younger women, consistent with the literature. ${ }^{[20,25]}$

Multiple logistic regression analysis adjusted for potential confounding and mediating factors, including parity, ART pregnancies, multiple pregnancies, and major fetal abnormalities, demonstrated that VAMA is an independent risk factor for adverse pregnancy outcomes, including an increased risk of GDM, polyhydramnios, cesarean section, and adverse neonatal outcomes, including a higher risk of stillbirths, preterm delivery, lower birth weight, lower 5-minute Apgar scores, and NICU admission. It means that the association between VAMA and these adverse pregnancy outcomes markedly persists after adjusting for the abovementioned pregnancy-related characteristics in the multiple regression analysis. Thus, we indicate an increased risk of experiencing an adverse pregnancy outcome for groups of pregnant women at VAMA. These results should be taken into account when counseling and managing pregnant women of VAMA.

The main strength of this study is the large sample size relative to previously published studies with low sample sizes. We also examined both maternal and neonatal data, which provide us to perform univariate and multivariate analyses on both maternal and neonatal confounding variables that might have affected the study results. However, there are some limitations to this study. This study has been organized retrospectively and may contain limitations of this kind studies. Since our hospital is a tertiary referral center, the incidence of adverse pregnancy outcomes may be higher than in the entire population.

\section{Conclusion}

Our study demonstrates a strong and significant association between VAMA and adverse pregnancy outcomes, including an increased risk of gestational diabetes mellitus, polyhydramnios, cesarean section, and adverse neonatal outcomes, including a higher risk of stillbirths, preterm delivery, lower birth weight, lower 5-minute Apgar scores, and NICU admission. Patients should be informed that the probability of pregnancy complications and adverse neonatal outcomes with rises in age. Also, these patients should be managed with appropriate care protocols and antenatal care services should be optimized for pregnant women with VAMA.

Funding: This work did not receive any specific grant from funding agencies in the public, commercial, or not-for-profit sectors.

Compliance with Ethical Standards: The authors stated that the standards regarding research and publication ethics, the Personal Data Protection Law and the copyright regulations applicable to intellectual and artistic works are complied with and there is no conflict of interest.

\section{References}

1. Schmidt L, Sobotka T, Bentzen JG, Nyboe Andersen A; ESHRE Reproduction and Society Task Force. Demographic and medical consequences of the postponement of parenthood. Hum Reprod Update 2012;18:29-43. [PubMed] [CrossRef]

2. Matthews TJ, Hamilton BE. First births to older women continue to rise. NCHS Data Brief 2014;(152):1-8. [PubMed]

3. Turkey Statistical Institute. Birth statistics 2019 [Internet]. Ankara: Turkey Statistical Institute. [cited 2020 Jul 6]. Available from https://tuikweb.tuik.gov.tr/PreHaberBultenleri.do?id=33707

4. Balasch J, Gratacós E. Delayed childbearing: effects on fertility and the outcome of pregnancy. Curr Opin Obstet Gynecol 2012;24:187-93. [PubMed] [CrossRef]

5. Attali E, Yogev Y. The impact of advanced maternal age on pregnancy outcome. Best Pract Res Clin Obstet Gynaecol 2021; 70:2-9. [PubMed] [CrossRef]

6. Sauer MV. Reproduction at an advanced maternal age and maternal health. Fertil Steril 2015;103:1136-43. [PubMed] [CrossRef]

7. Hsieh TT, Liou JD, Hsu JJ, Lo LM, Chen SF, Hung TH. Advanced maternal age and adverse perinatal outcomes in an Asian population. Eur J Obstet Gynecol Reprod Biol 2010;148: 21-6. [PubMed] [CrossRef]

8. Klemetti R, Gissler M, Sainio S, Hemminki E. At what age does the risk for adverse maternal and infant outcomes increase? Nationwide register-based study on first births in Finland in 2005-2014. Acta Obstet Gynecol Scand 2016;95: 1368-75. [PubMed] [CrossRef] 
9. Nilsen ABV, Waldenström U, Hjelmstedt A, Rasmussen S, Schytt E. Characteristics of women who are pregnant with their first baby at an advanced age. Acta Obstet Gynecol Scand 2012;91:353-62x. [PubMed] [CrossRef]

10. Khalil A, Syngelaki A, Maiz N, Zinevich Y, Nicolaides KH. Maternal age and adverse pregnancy outcome: a cohort study. Ultrasound Obstet Gynecol 2013;42:634-43. [PubMed] [CrossRef]

11. Frederiksen LE, Ernst A, Brix N, Braskhøj Lauridsen LL, Roos $\mathrm{L}$, Ramlau-Hansen $\mathrm{CH}$, et al. Risk of adverse pregnancy outcomes at advanced maternal age. Obstet Gynecol 2018;131: 457-63. [PubMed] [CrossRef]

12. ACOG Practice Bulletin No. 202: Gestational hypertension and preeclampsia. Obstet Gynecol 2019;133:e1-e25. [PubMed] [CrossRef]

13. International Association of Diabetes and Pregnancy Study Groups Consensus Panel; Metzger BE, Gabbe SG, Persson B, Buchanan TA, Catalano PA, Damm P, et al. International association of diabetes and pregnancy study groups recommendations on the diagnosis and classification of hyperglycemia in pregnancy. Diabetes Care 2010;33:676-82. [PubMed] [CrossRef]

14. Behram M, Oğlak SC, Başkıran Y, Süzen Çaypınar S, Akgöl S, Tunç Ş, et al. Maternal serum IL-22 concentrations are significantly upregulated in patients with preterm premature rupture of membranes. Ginekol Pol 2021;91:631-6. [PubMed] [CrossRef]

15. Arthuis C, Diguisto C, Lorphelin H, Dochez V, Simon E, Perrotin F, et al. Perinatal outcomes of intrahepatic cholestasis during pregnancy: an 8-year case-control study. PLoS One 2020;15(2):e0228213. [PubMed] [CrossRef]

16. Oglak SC, Obut M, Tahaoglu AE, Demirel NU, Kahveci B, Bagli I. A prospective cohort study of shock index as a reliable marker to predict the patient's need for blood transfusion due to postpartum hemorrhage. Pak J Med Sci 2021;37:863-8. [PubMed] [CrossRef]

17. Fishel Bartal M, Chen HY, Blackwell SC, Chauhan SP, Sibai BM. Neonatal morbidity in late preterm small for gestational age neonates. J Matern Fetal Neonatal Med 2021;34:3208-13. [PubMed] [CrossRef]

18. Oğlak SC, Bademkıran MH, Obut M. Predictor variables in the success of slow-release dinoprostone used for cervical ripening in intrauterine growth restriction pregnancies. J Gynecol Obstet Hum Reprod 2020;49:101739. [PubMed] [CrossRef]

19. Behram M, Oğlak SC, Dağ İ. Circulating levels of Elabela in pregnant women complicated with intrauterine growth restriction. J Gynecol Obstet Hum Reprod 2021;50:102127. [PubMed] [CrossRef]
20. Ates S, Batmaz G, Sevket O, Molla T, Dane C, Dane B. Pregnancy outcome of multiparous women aged over 40 years. Int J Reprod Med 2013;2013:287519. [PubMed] [CrossRef]

21. Schimmel MS, Bromiker R, Hammerman C, Chertman L, Ioscovich A, Granovsky-Grisaru S, et al. The effects of maternal age and parity on maternal and neonatal outcome. Arch Gynecol Obstet 2015;291:793-8. [PubMed] [CrossRef]

22. Marozio L, Picardo E, Filippini C, Mainolfi E, Berchialla P, Cavallo F, et al. Maternal age over 40 years and pregnancy outcome: a hospital-based survey. J Matern Fetal Neonatal Med 2019;32:1602-8. [PubMed] [CrossRef]

23. Bruno RM, Masi S, Taddei M, Taddei S, Virdis A. Essential hypertension and functional microvascular ageing. High Blood Press Cardiovasc Prev 2018;25:35-40. [PubMed] [CrossRef]

24. Cakmak Celik F, Aygun C, Kucukoduk S, Bek Y. Maternal and neonatal outcomes in advanced maternal age: a retrospective cohort study. J Matern Fetal Neonatal Med 2017;30:2452-6. [PubMed] [CrossRef]

25. Arya S, Mulla ZD, Plavsic SK. Outcomes of women delivering at very advanced maternal age. J Womens Health (Larchmt) 2018;27:1378-84. [PubMed] [CrossRef]

26. Kanmaz AG, İnan AH, Beyan E, Ögür S, Budak A. Effect of advanced maternal age on pregnancy outcomes: a single-centre data from a tertiary healthcare hospital. J Obstet Gynaecol 2019;39:1104-11. [PubMed] [CrossRef]

27. Fitzpatrick KE, Tuffnell D, Kurinczuk JJ, Knight M. Pregnancy at very advanced maternal age: a UK populationbased cohort study. BJOG 2017;124:1097-106. [PubMed] [CrossRef]

28. Saleh Gargari S, Seify Z, Haghighi L, Khoshnood Shariati M, Mirzamoradi M. Risk factors and consequent outcomes of placenta previa: report from a referral center. Acta Med Iran 2016; 54:713-7. [PubMed]

29. Yogev Y, Melamed N, Bardin R, Tenenbaum-Gavish K, BenShitrit G, Ben-Haroush A. Pregnancy outcome at extremely advanced maternal age. Am J Obstet Gynecol 2010;203:558. e1-7. [PubMed] [CrossRef]

30. Lao TT, Sahota DS, Cheng YK, Law LW, Leung TY. Advanced maternal age and postpartum hemorrhage - risk factor or red herring? J Matern Fetal Neonatal Med 2014;27: 243-6. [PubMed] [CrossRef]

31. Flenady V, Middleton P, Smith GC, Duke W, Erwich JJ, Khong TY, et al.; Lancet's Stillbirths Series Steering Committee. Stillbirths: the way forward in high-income countries. Lancet 2011;377(9778):1703-17. [PubMed] [CrossRef]

32. Huang L, Sauve R, Birkett N, Fergusson D, van Walraven C. Maternal age and risk of stillbirth: a systematic review. CMAJ 2008;178:165-72. [PubMed] [CrossRef]

Publisher's Note: The content of this publication does not necessarily reflect the views or policies of the publisher, nor does any mention of trade names, commercial products, or organizations imply endorsement by the publisher. Scientific and legal responsibilities of published manuscript belong to their author(s). The publisher remains neutral with regard to jurisdictional claims in published maps and institutional affiliations. 